\title{
Constraints on the bulk Lorentz factor of gamma-ray bursts with the detection rate by Fermi LAT
}

\author{
Ye Chen, ${ }^{1,2}$ Ruo-Yu Liu ${ }^{3,4}$ and Xiang-Yu Wang ${ }^{1,2}$ \\ ${ }^{1}$ School of Astronomy and Space Science, Nanjing University, Nanjing 210093, China \\ ${ }^{2}$ Key Laboratory of Modern Astronomy and Astrophysics (Nanjing University), Ministry of Education, Nanjing 210093, China \\ ${ }^{3}$ Max-Planck-Institut für Kernphysik, Heidelberg D-69117, Germany \\ ${ }^{4}$ Deutsches Elektronen Synchrotron (DESY), Platanenallee 6, Zeuthen D-15738, Germany
}

Accepted 2018 April 29. Received 2018 April 6; in original form 2017 June 5

\begin{abstract}
The bulk Lorentz factor $(\Gamma)$ of the outflow is an essential parameter for understanding the physics of gamma-ray burst (GRB). Informations about the Lorentz factors of some individual GRBs have been obtained from the spectral features of the high-energy gamma-ray emissions $(>100 \mathrm{MeV})$, assuming that the spectral breaks or cutoffs are due to the pair-production attenuation (i.e. $\gamma \gamma \rightarrow \mathrm{e}^{+} \mathrm{e}^{-}$). In this paper, we attempt to interpret the dependence of the Large Area Telescope (LAT) detection rate of GRBs on the number of high-energy gamma-rays, taking into account the attenuation effect. We first simulate a long-GRB sample with Monte Carlo method using the luminosity function, rate distribution with redshift, and properties of the GRB spectrum. To characterize the distribution of the Lorentz factors, we assume that the Lorentz factors follow the relation $\Gamma=\Gamma_{0} E_{\text {iso }, 52}^{k}$, where $E_{\text {iso, } 52}$ is the isotropic photon energy in unit of $10^{52} \mathrm{erg}$. After taking into account the attenuation effect related with the above Lorentz factor distribution, we are able to reproduce the LAT-detected rate of GRBs as the function of the number of gamma-rays for suitable choice of the values of $\Gamma_{0}$ and $k$. The result suggests that the distribution of the bulk Lorentz factor for the majority of GRBs is in the range of $50-250$.
\end{abstract}

Key words: radiation mechanisms: non-thermal-gamma-rays: general.

\section{INTRODUCTION}

Detection of high-energy gamma-rays from gamma-ray burst (GRBs) requires that these photons $(\gg \mathrm{MeV})$ escape the source without suffering from attenuation due to pair production $\left(\gamma \gamma \rightarrow \mathrm{e}^{+} \mathrm{e}^{-}\right)$(e.g. Krolik \& Pier 1991; Fenimore, Epstein \& Ho 1993; Woods \& Loeb 1995; Baring \& Harding 1997). Requiring that the absorption optical depth $\tau_{\gamma \gamma} \lesssim 1$ within the source for highenergy photons, one can deduce a lower limit on the bulk Lorentz factor $(\Gamma)$ of the emitting region, which is usually $\gtrsim 100$ (Lithwick \& Sari 2001). Recently, thanks to wide energy coverage and good sensitivity of Large Area Telescope (LAT) on Fermi, the spectral cutoffs or breaks have been observed in several GRBs (Ackermann et al. 2011; Tang et al. 2015; Vianello et al. 2017; Yassine et al. 2017). Assuming that the spectral breaks are caused by the attenuation effect, the bulk Lorentz factors of these GRBs are obtained (e.g. Ackermann et al. 2011), which are in the rage of 200-1000. However, for the vast majority of GRBs, we still do not know the values of their bulk Lorentz factors.
Using the GRB sample detected in the first $3 \mathrm{yr}$ by Fermi/LAT, Ackermann et al. (2013) obtained the detected number of GRBs as a function of the number of gamma-rays. The detection rate by Fermi/LAT is about $8 \mathrm{yr}^{-1}$, which is smaller than the expected one derived from the extrapolation of the empiric Band function to high energy (Band et al. 2009). Additionally, the difference between the predicted and observed numbers of GRBs increases for bursts with increasing number of gamma-rays in the LAT data. The lack of GRBs detected by Fermi/LAT has been interpreted as arising from the attenuation effect that suppress the high-energy emission (Guetta, Pian \& Waxman 2011). To consider the attenuation in each GRB, we assume that the Lorentz factor follows the relation $\Gamma \propto E_{\text {iso }}^{k}$, motivated by some recent studies (Liang et al. 2010; Ghirlanda et al. 2012; Tang et al. 2015). In this paper, we attempt to interpret the dependence of the number of detected GRBs on the number of gamma-rays by considering the attenuation effect. As this deals with the whole population of GRBs, such an approach could reveal the information about the Lorentz factors of the major population of GRBs.

Our paper is organized as follows. In Section 2, we first simulate a sample of GRBs according to the luminosity function, the event rate distribution with redshift, and the properties of prompt GRB 
spectrum. Then in Section 3, we consider the attenuation effect on the spectra of high-energy gamma-rays and try to reproduce the relation between the number of GRBs detected per year and the number of gamma-rays detected in one GRB. Then we give our conclusion and discussion in Section 4.

\section{SIMULATION OF A GRB SAMPLE}

In this work, we assume that the distributions of GRBs in luminosity and in redshift are independent. We adopt the luminosity function and the redshift evolution function of GRBs given by Wanderman \& Piran (2010), which are obtained directly from the redshift-luminosity distribution of long GRBs detected by Swift, i.e.

$\phi\left(L_{\text {iso }}\right)=\left\{\begin{array}{l}\left(\frac{L_{\text {iso }}}{L_{b}}\right)^{-a_{1}}, L_{\text {iso }}<L_{b} \\ \left(\frac{L_{\text {iso }}}{L_{b}}\right)^{-a_{2}}, L_{\text {iso }} \geq L_{b}\end{array}\right.$

and

$\rho(z)=\rho_{0} \begin{cases}(1+z)^{n_{1}}, & z<z_{b} \\ (1+z)^{n_{2}}\left(1+z_{b}\right)^{n_{1}-n_{2}}, & z \geq z_{b},\end{cases}$

where $a_{1}=1.17, a_{2}=2.44, L_{b}=10^{52.5} \mathrm{erg} \mathrm{s}^{-1}, n_{1}=2.1, n_{2}=-1.4$, $z_{b}=3.1$, and $\rho_{0}=1.3 \mathrm{Gpc}^{-3} \mathrm{yr}^{-1}$. Here $L_{\text {iso }}$ is the peak bolometric luminosity of GRB in $1-10000 \mathrm{keV}$. Setting $L_{\text {iso }}$ from $10^{50}$ to $10^{54} \mathrm{erg} \mathrm{s}^{-1}$ and the redshift $z \leq 8$, we use a Monte Carlo simulation to generate a sample of GRBs with luminosity and redshift following equations (1) and (2). The generated luminosity distribution and the redshift distribution are shown in Fig. 1. We then obtain the isotropic-equivalent photon energy of each GRB following the relation between the luminosity and the total energy given by Ghirlanda et al. (2012), i.e.

$\log E_{\text {iso }, 52}=1.07 \log L_{\text {iso }, 52}+(0.66 \pm 0.54)$.

As is well accepted, the Band function (Band et al. 1993) can describe the photon spectra of GRBs as

$N(E)=N_{0} \begin{cases}\left(\frac{E}{E_{0}}\right)^{\alpha} \mathrm{e}^{-E / E_{0}}, & E<(\alpha-\beta) E_{0} \\ \left(\frac{E}{E_{0}}\right)^{\beta}(\alpha-\beta)^{\alpha-\beta} \mathrm{e}^{\beta-\alpha}, & E \geq(\alpha-\beta) E_{0},\end{cases}$

where $N_{0}$ is a normalization constant. Here $\alpha$ and $\beta$ are the photon indexes below and above the break energy $(\alpha-\beta) E_{0}$, respectively. We generate their values based on the statistical distribution of these two quantities obtained from Fermi/GBM GRBs of the first $2 \mathrm{yr}$ (Goldstein et al. 2012), ${ }^{1}$ as shown in Fig. 2. The peak energy in the energy spectrum is related with $E_{0}$ by $E_{\text {peak }}=(2+\alpha) E$ for GRBs with $\beta_{0}<-2$ (which is usually the case). So, to obtain $E_{0}$ what we need is $E_{\text {peak }}$ for each GRB.

To determine $E_{\text {peak }}$ of each GRBs, we study the relation between $E_{\text {peak }}$ and the burst bolometric fluence, ${ }^{2}$ following the treatment of

\footnotetext{
${ }^{1}$ Note that there is a peak around -6 in the distribution of $\beta$. This is because Goldstein et al. (2012) put all the GRBs with $\beta<-6$ into the bin of -6 . So the function used to fitting the distribution, of course, is not a realistic distribution for $\beta$ at the very soft side. But adopting this function will not introduce error in our simulation, because extrapolating the spectrum to $>100 \mathrm{MeV}$ with $\beta=-6$ will already result in a very low flux of the GRB at high energies, with no chance of being detected by LAT. It makes no difference if we extrapolate the spectrum with a smaller $\beta$.

${ }^{2}$ Here, one can also find $E_{\text {peak }}$ of each GRB, in principle, through some empirical $E_{\text {peak }}-L_{\text {iso }}$ and $E_{\text {peak }}-E_{\text {iso }}$ relations (e.g. Amati et al. 2002; Ghirlanda et al. 2012) which is usually adopted in previous studies (e.g. Kakuwa et al.
}

Lloyd, Petrosian \& Mallozzi (2000), Ghirlanda et al. (2008), and Nava et al. (2008), but with the latest Fermi-GBM sample of 72 long GRBs with measured redshifts (see Table A1). We extrapolate the measured fluences in the energy range of 10-1000 to $1-10000 \mathrm{keV}$, in order to comply with the energy range used for the luminosity function. We find that there is a good correlation between $E_{\text {peak }}$ and the fluence $F$ (see Fig. 3), which is, with 95 percent confidence bounds,

$\log \left(\frac{E_{\text {peak }}}{\mathrm{keV}}\right)=(0.28 \pm 0.04) \log \left(\frac{F}{\mathrm{erg} \mathrm{cm}^{-2}}\right)+(3.56 \pm 0.21)$.

Here both $E_{\text {peak }}$ and the fluence are the values in the observer's frame. Based on the above relation, the peak energy of each generated GRB in our sample can be obtained from the fluence which is determined by the generated $E_{\text {iso }}$ and redshift $z$. We set the trigger threshold of GRB detector in our simulation to be 0.74 photons $\mathrm{cm}^{-2} \mathrm{~s}^{-1}$ in $50-300 \mathrm{keV}$, the same as that of $\mathrm{GBM}$ (Meegan et al. 2009). Finally, we generate a sample of 1000 triggered GRBs, and compare the fluence distribution (in the energy range of $10-1000 \mathrm{keV}$ ) and $E_{\text {peak }}$ distribution of the simulated GRB sample to those in the observed sample, respectively, in Fig. 4. The K-S tests return an accepting probability of 15 percent and 22 per cent, respectively, for fluence distribution and for $E_{\text {peak }}$ distribution.

\section{CONSTRAINTS ON THE BULK LORENTZ FACTORS OF GRBS}

\subsection{Extension of the Band function to high energy}

The properties of the generated GRBs include isotropic luminosity in $1-10000 \mathrm{keV}$, isotropic photon energy in $1-10000 \mathrm{keV}$, redshift, spectral indices, and peak energy of each GRB, based on which one can get differential fluence of the GRB at the Earth. We extrapolate the fluence of each GRB up to $100 \mathrm{GeV}$ to obtain the high-energy photon flux at Earth (in unit of photons $\mathrm{cm}^{-2}$ ) if there is no absorption, and calculate the expected number of high-energy photons (100 MeV to $100 \mathrm{GeV}$ ) detected by LAT. We treat the effective area of LAT as a product of two independent functions of photon energy and incidence angle, respectively, where the former function follows the response of the effective area to energy at normal incidence $(\theta=0)$ and the latter one follows the response of the effective area to incidence angle for $10 \mathrm{GeV}$ photons. Based on the assumption of an isotropic distribution of GRBs in the celestial sphere, we generate the incidence angle for each GRB. A GRB is regarded as being detected by LAT if its $100 \mathrm{MeV}$ to $100 \mathrm{GeV}$ flux at the Earth can lead to detection of $\geq 5$ events at LAT. Given all these necessary information (the effective area of LAT, the spectrum of simulated GRBs, and their incidence angle), we find that 145 GRBs in our sample can be detected by LAT, which translates to a detection rate of $\sim 29$ GRBs per year. This means that $\sim 15$ per cent of the total triggered GRBs would be detected by LAT, while the observed rate is only about 5 percent (Ackermann et al. 2013). We also calculate the expected number of GRBs detected by LAT per year as a function of the number of $>100 \mathrm{MeV}$ photons detected, as shown

2012; Liu \& Wang 2013). However, the generated $E_{\text {peak }}$ distribution in our sample with such relations is rejected by $\mathrm{K}-\mathrm{S}$ test when compared with the $E_{\text {peak }}$ distribution of observed GRBs. Therefore, we use an empirical $E_{\text {peak }}-$ fluence relation instead. 

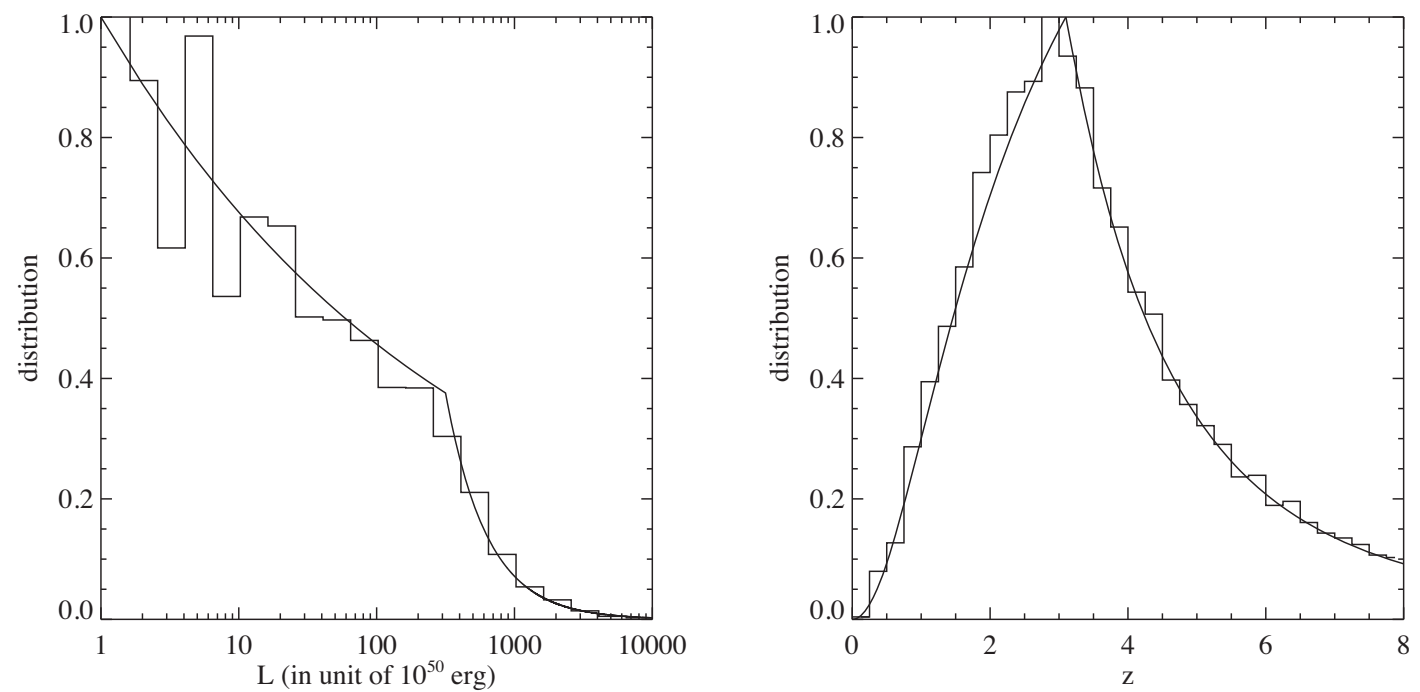

Figure 1. The normalized distribution of GRBs appearing with a bolometric luminosity $L$ (left-hand panel) and appearing at redshift $z$ (right-hand panel). The solid curves are the theoretical distribution based on the luminosity function and the redshift evolution function. For luminosity distribution, the solid curve $\propto \varphi(L)$ while for redshift distribution, the solid curve $\propto \rho(z) 4 \pi D_{c}(z)^{2} /(1+z) / H(z)$ with $D_{c}$ the comoving distance at $z$ and $H(z)$ the Hubble parameter at $z$. The histograms are the distributions of luminosity $L$ and redshift $z$ of the total simulated GRBs. Note that not all the simulated GRBs can trigger GBM, so the luminosity distribution and the redshift distribution of triggered GRBs should be different from those shown here.
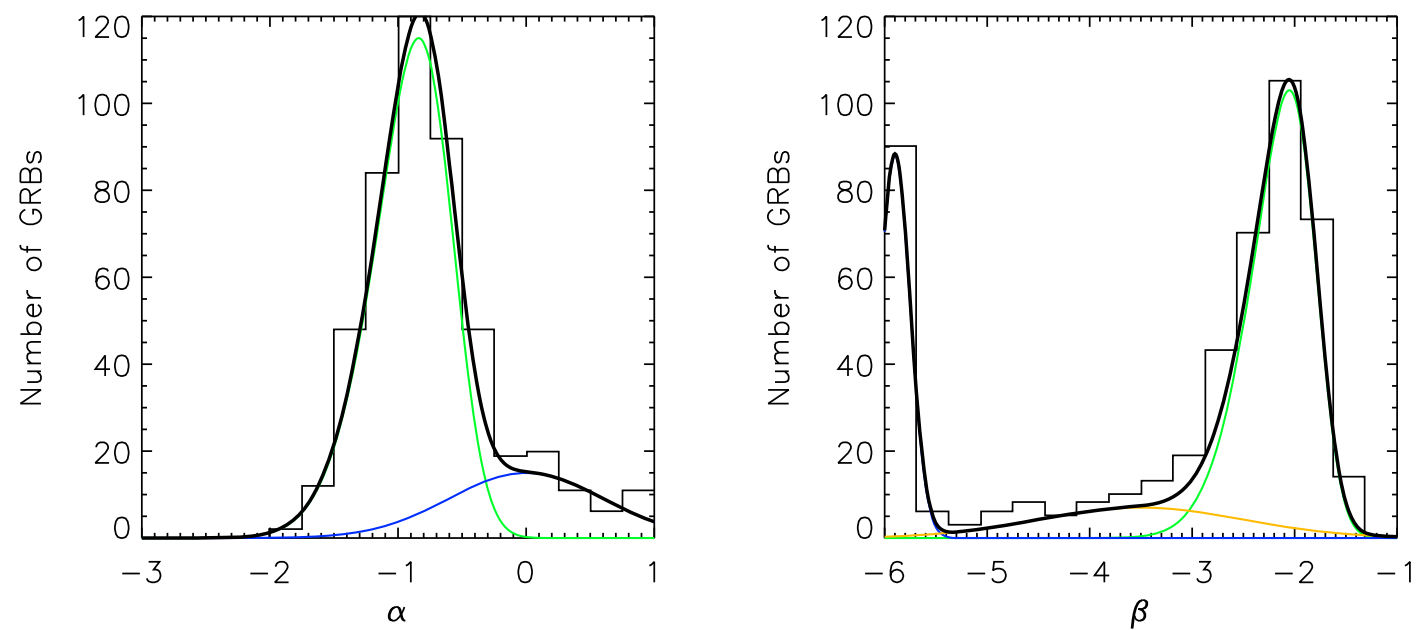

Figure 2. Distributions of the low-energy spectral index $\alpha$ (left) and high-energy spectral index $\beta$ (right) from fluence spectral fits. The histograms, which are taken from Goldstein et al. (2012), represent the distributions of the observed GRB sample. Our fitting to the observed distributions are shown as solid curves. The distribution of $\alpha$ is fitted by the superposition (black curve) of a Gaussian distribution with the mean value of 0 and deviation of 0.6 (blue) and a skew normal distribution with the mean value of -0.6 , deviation of 0.45 , and skewness of -2 (green). The distribution of $\beta$ is fitted by the superposition (black curve) of a Gaussian distribution with the mean value of -5.9 and deviation of 0.15 (blue), a Gaussian distribution with the mean value of -3.5 and deviation of 1 (yellow) and a skew normal distribution of the mean value of -1.8 , deviation of 0.5 , and skewness of -2.5 (green). These functions are used to generate the values of $\alpha$ and $\beta$ for our simulated GRB sample.

by the red dashed line in each graph of Figs 5 and 6 . It is significantly larger than the observed rate, which is shown by the black dashed line. Such an inconsistency between the simulated sample and the observed sample may imply an internal absorption of those high-energy photons, as we will discuss below.

\subsection{Internal absorption}

The pair production process $\left(\gamma \gamma \rightarrow \mathrm{e}^{-} \mathrm{e}^{+}\right)$is relevant for highenergy photons in a radiation field of compact astrophysical objects, leading to the possible attenuation of high-energy photons. This process has a kinematic threshold which is given by $s \equiv \epsilon \epsilon_{1}(1-\cos \theta)$, with $\epsilon$ and $\epsilon_{1}$ being the energies of soft target photon and high-energy incidence photon, respectively, in units of $m_{e} c^{2}$ while $\theta$ is the colliding angle of the two photons (in the lab frame). During the prompt emission phase of a GRB, a huge amount of $\mathrm{X} / \gamma$-ray photons are produced in the compact fireball, which may in principle lead to absorption to the $\mathrm{GeV}$ photons. However, the ultrarelativistic bulk motion of the fireball would result in nearly parallel propagation of all the emitted photons (i.e. a large $\cos \theta$ ) and hence suppress the interaction or the optical depth of GeV photons. Thus, the detection rate of $\mathrm{GeV}$ emissions from GRBs may give a clue to the bulk Lorentz factor of the fireball. We note that the extragalactic background light (EBL) will also attenuate $>10 \mathrm{GeV}$ 


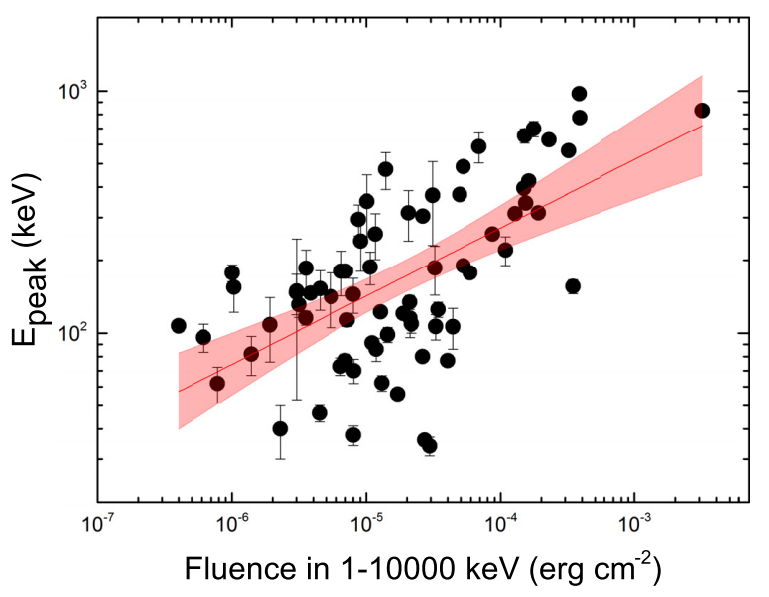

Figure 3. Peak energy $\left(E_{\text {peak }}\right)$ as a function of bolometric fluence $F$. The solid line shows the least-squares fit with a power law to the $E_{\text {peak }}-F$ correlation to the sample of $72 \mathrm{Fermi} / \mathrm{GBM}$ GRBs with measured redshift (filled blocks), while the shadow shows the 95 per cent C.L. bound.

photons when these photons are propagating in the intergalactic space. We ignore this effect in this work because most of highenergy photons of GRBs detected by LAT are around hundreds of $\mathrm{MeV}$, given that the high-energy spectral indices of most GRBs (i.e. $\beta$ ) are less than -2 . Hence, the high-energy photon detection rate of LAT would be barely changed whether we consider the attenuation by EBL or not.

Considering the high-energy photons are emitted on-axis and all the photons are emitted from the same radius, the $\gamma \gamma$ optical depth can be given by (Granot, Cohen-Tanugi \& Silva 2008; Hascoët et al. 2012)

$$
\begin{aligned}
\tau_{\gamma \gamma}\left(\epsilon_{1}\right)= & 2^{1+2 \beta} I(\beta) \frac{(-2-\beta) \sigma_{\mathrm{T}} L_{\gamma}\left(\epsilon>E_{\text {peak }}\right) \Delta T \Gamma^{2(1+\beta)}}{4 \pi R^{2} E_{\text {peak }}(1+z)} \\
& \times\left[\frac{m_{e}^{2} c^{4}}{\epsilon_{1} E_{\text {peak }}(1+z)^{2}}\right]^{1+\beta},
\end{aligned}
$$

where $\epsilon_{1}$ is the energy of an observed high-energy photon, $\sigma_{\mathrm{T}}$ is the Thomson cross-section. $I(\beta)=\int_{0}^{1} y g(y) \mathrm{d} y /\left(1-y^{2}\right)^{2+\beta}$ with $g(y)=\frac{3}{16}\left(1-y^{2}\right)\left[\left(3-y^{4}\right) \ln \frac{1+y}{1-y}-2 y\left(2-y^{2}\right)\right]$. Here we assume that only photons with energy beyond the spectral $E_{\text {peak }}$ are energetic enough to annihilate with high-energy photons, and hence the lowenergy spectral slope $\alpha$ does not show up in the expression. The radius of the fireball is given by $R=2 \Gamma^{2} c \Delta T$ with $\Delta T$ being the variability time-scale of a single pulse. We can see the value of $\tau_{\gamma \gamma}$ is sensitively dependent on $\Gamma$, i.e. $\tau_{\gamma \gamma} \propto \Gamma^{2 \beta-2}$, given $\beta<-2$ in most cases. Note that this formula gives a smaller optical depth than that obtained from the simple model assuming an isotropic radiation field in the comoving frame of the fireball. If one further considers that photons are emitted at different radius, the obtained optical depth will further decrease, as is indicated in previous studies (e.g. Granot et al. 2008; Zou, Fan \& Piran 2011; Hascoët et al. 2012).

The bulk Lorentz factor of GRB fireball is thought to be related with the isotropic luminosity or energy (Liang et al. 2010; Ghirlanda et al. 2012; Lü et al. 2012; Tang et al. 2015). We employ a relation given by $\Gamma=\Gamma_{0} E_{\text {iso, } 52}^{k}$, with $\Gamma_{0}$ and $k$ being free parameters, instead of using the relations obtained in the previous literatures which have large uncertainties. Here, we consider $k=0$ as the lower limit of $k$, since the previous studies all indicate a positive relation between the total energy and the bulk Lorentz factor. Then, we obtain optical depths for high-energy photons in each simulated GRB, and the flux after absorption can be given by $f_{\text {abs }}\left(\epsilon_{1}\right)=f\left(\epsilon_{1}\right) \exp ^{-\tau\left(\epsilon_{1}\right)}$. By tuning the values of $k$ and $\Gamma_{0}$, we can obtain the predicted LAT detection rate that matches the observed one. The feasible combinations of $\Gamma_{0}$ and $k$ are so many that we cannot exhaust them. Thus, we present the LAT detection rate for three representative combinations of $\Gamma_{0}$ and $k$ in the case of $\Delta T=100 \mathrm{~ms}$ in Fig. 5. The black dashed lines represent the observed rate and the red dashed lines represent the case without considering $\gamma \gamma$ absorption. Solid lines of different colours represent the results after considering the absorption for different values of $k$, while the black solid lines represent the solutions. In general, we see that the solid lines approach the red dashed lines (the no absorption case) more closely for larger $\Gamma_{0}$, implying that the absorption is less important for larger $\Gamma_{0}$, as it leads to a larger dissipation radius and consequently a smaller density of target photons. On the other hand, a larger $k$ also leads to less absorption, because the energetic GRBs $\left(E_{\text {iso }}>10^{52} \mathrm{erg}\right)$ will then have larger Lorentz factors and, in consequence, smaller opacities. A larger $k$, in the meantime, results in smaller Lorentz factors for less energetic $\left(E_{\text {iso }}<10^{52} \mathrm{erg}\right) \mathrm{GRBs}$, which results in stronger internal absorption. But these 'dim' GRBs are mostly un-detectable to LAT unless appearing in our vicinity, so the high opacity does not affect the LAT detection rate for these dim GRBs. The feasible range for $k$ is about $k<0.3$. A larger $k$ will result in a small optical depth for high-luminosity GRBs and overpredict the LAT detection. Although the optical depth can be increased by choosing a small $\Gamma_{0}$, the optical depth for $\lesssim 10 \mathrm{MeV}$ photons in low-luminosity GRBs would then be larger than unity, which is not consistent with observation. This sets a lower bound for $\Gamma_{0}$ to be about 50 . The upper bound of $\Gamma_{0}$ can be obtained with $k=0$. Given the uncertainty in the variability time-scale, we also consider a case with $\Delta=10 \mathrm{~ms}$ and the results are shown in Fig. 6. Given a smaller variability time, we need a larger bulk Lorentz factor to achieve the same opacity. However, since the opacity sensitively depends on the bulk Lorentz factor, the later one does not need to increase much.

In Fig. 7, we show the distributions of the Lorentz factors of simulated GRBs for the solutions of $\Gamma_{0}$ and $k$ that can reproduce the LAT detection rate with $\Delta T=100$ and $10 \mathrm{~ms}$, respectively, in the upper panel and the lower panel. Interestingly, we can see the Lorentz factors for most GRBs lie between 50 and 250 for all these solutions. We note that, however, some bright GRBs present a hard extra-component spectrum at $\mathrm{GeV}$ which is not considered in this work. The presence of the extra-component would increase the detectability, leading to the detection rate obtained here is a lower limit and the Lorentz factor is an upper limit. But the fraction of GRBs that have a significant extra component is small, so this correction should not be large. We also check the Thomson opacity for each GRBs. For the given combinations shown in Fig. 7. The Thomson optical depths for almost all the GRBs are smaller than unity, and 60-90 per cent GRBs have optical depth smaller than 0.1 in the case of $\Delta T=100 \mathrm{~ms}$ and that fraction in the case of $\Delta T=10 \mathrm{~ms}$ is $40-80$ per cent, unless the baryon loading factor is $\gg 1$. Thus, the Thomson opacity is less constraining than the $\gamma \gamma$ opacity on the bulk Lorentz factor.

\section{DISCUSSIONS AND CONCLUSIONS}

In this work, we studied the properties of the bulk Lorentz factors of GRBs from the viewpoint of the LAT detection rate. Taking advantage of various empirical relations of GRBs, we first generated a GRB sample with information about the burst distances and spectra. We then calculate the LAT detection rate as a function of the 

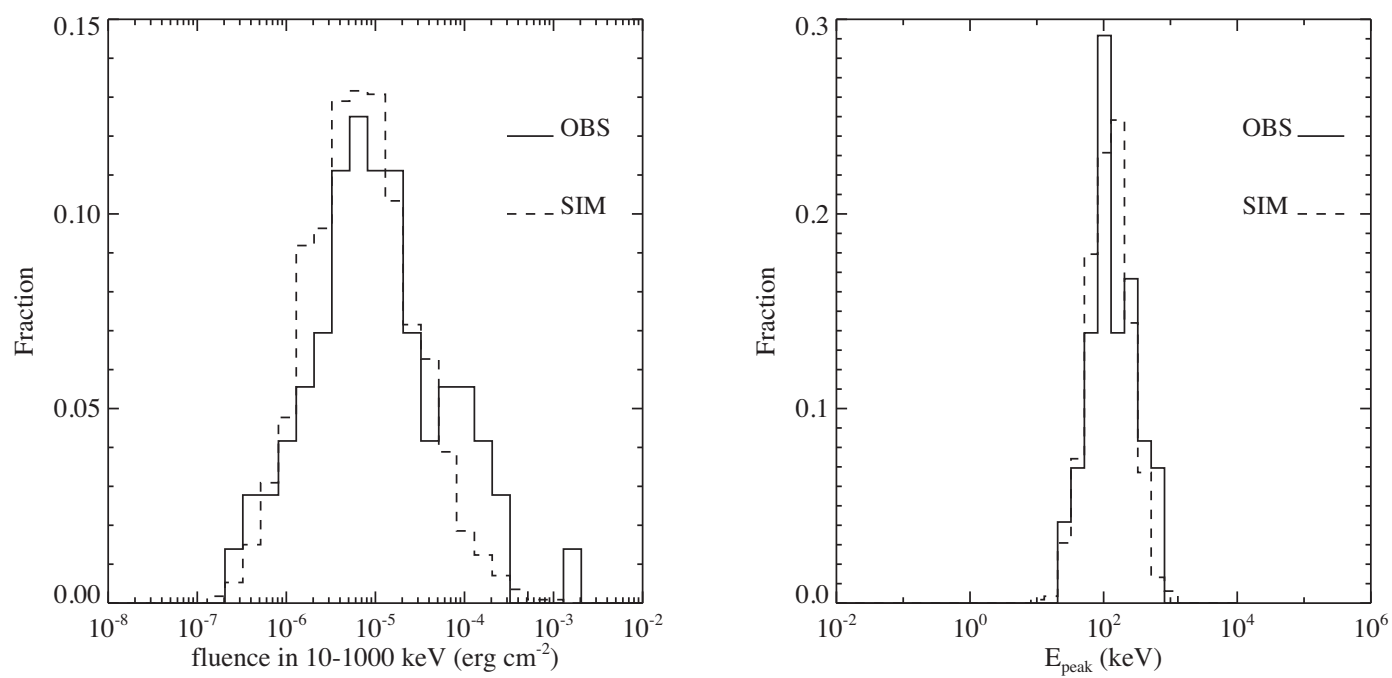

Figure 4. Left: Comparison of the fluence (in the energy range of 10-1000 keV) distributions of simulated GRBs (solid line) to that of Fermi/GBM GRBs with measured redshift (dashed line). The K-S test gives a probability of 15 percent for the agreement of the two distributions. Right: Same as the left-hand panel but for $E_{\text {peak }}$ distributions. The K-S test gives a probability of 22 per cent for the agreement of the two distributions.
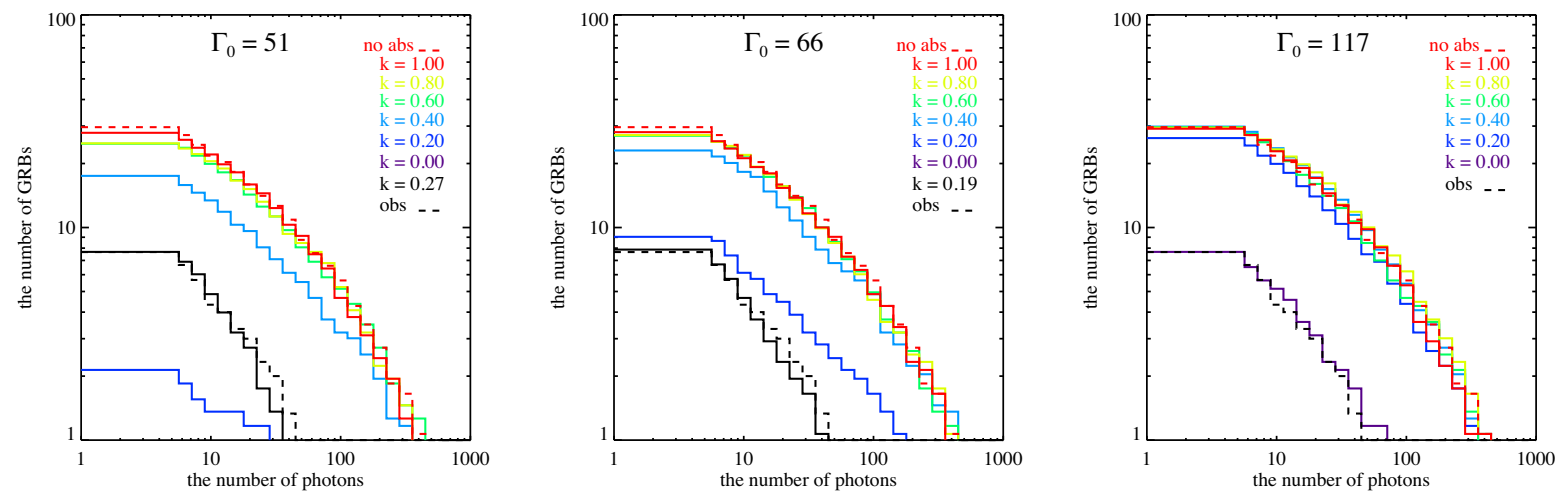

Figure 5. Predicted number of GRB detected by LAT per year as a function of the number of high-energy ( $>100 \mathrm{MeV})$ photons detected from each burst in the case of $\Delta t=100 \mathrm{~ms}$. Note that we set the detection threshold of LAT to be receiving $\geq 5$ high-energy photons from a GRB. $\Gamma_{0}$ is different in each panel. As shown, The black dashed lines represent the realistic LAT detection rate averaged over the period from 2008 August 25 to 2011 July 31 . The red dashed lines represent the case of no absorption. Solid lines with different colours represent cases for different values of $k$ and black solid lines represent the solutions that can match the observation.
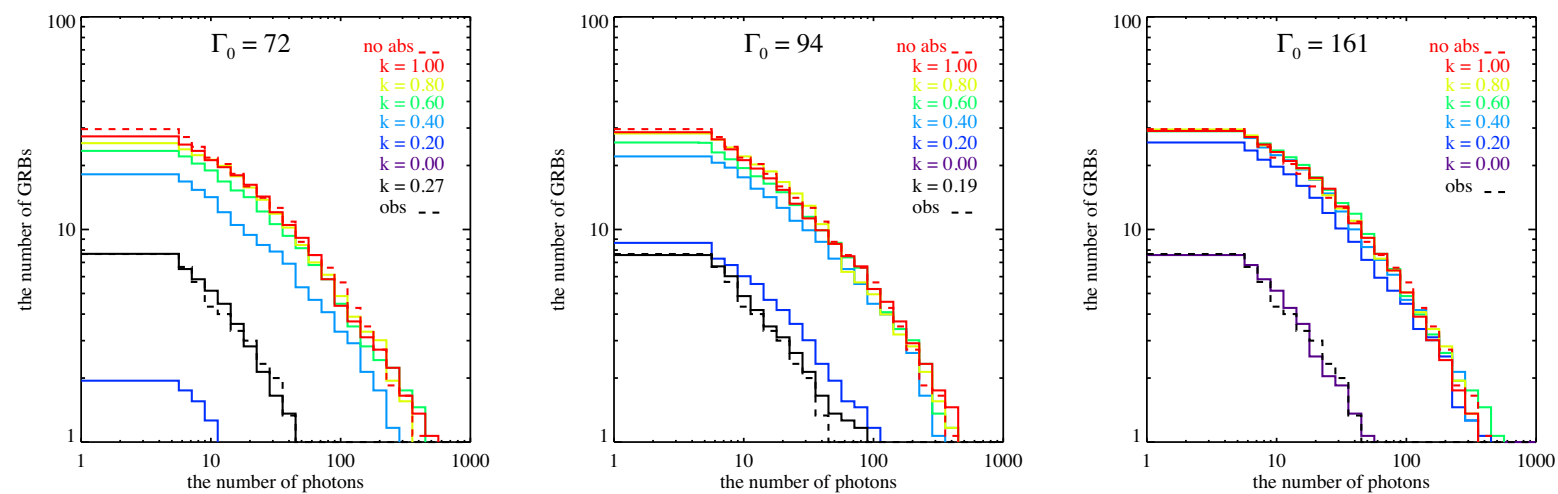

Figure 6. The same as Fig. 5 but for $\Delta t=10 \mathrm{~ms}$. 

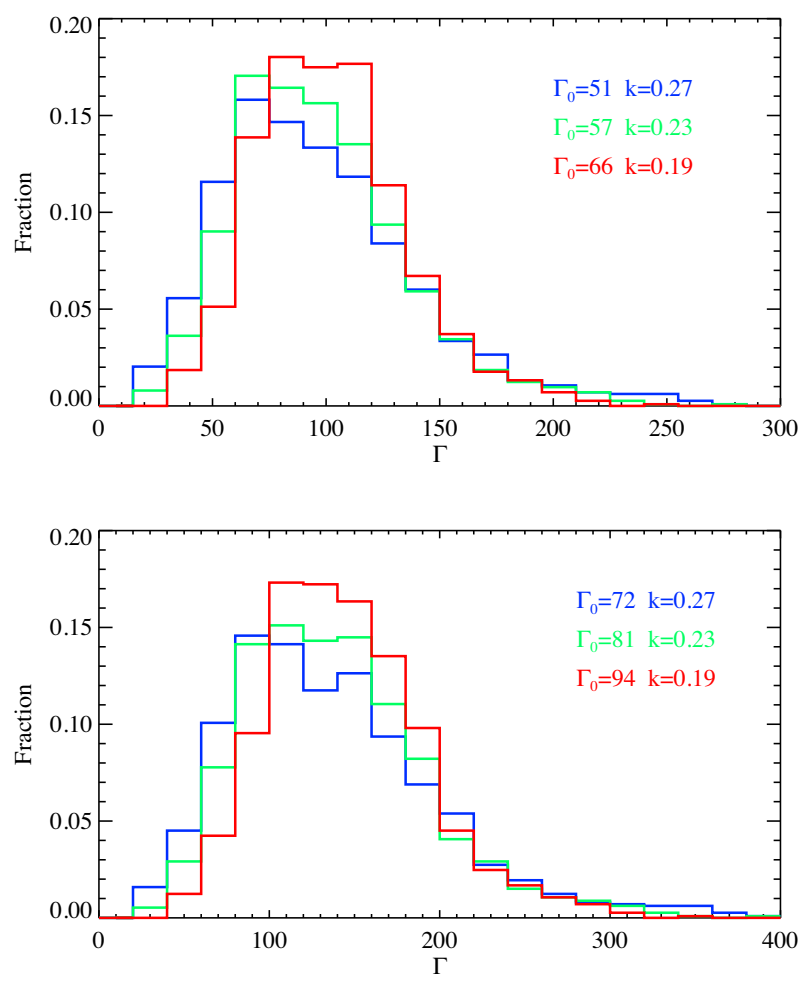

Figure 7. The number fraction of GRBs in the simulated sample as a function of the bulk Lorentz factors for various combinations of $\gamma_{0}$ and $k$ that can match the Fermi/LAT observation, in the case of $\Delta t=100 \mathrm{~ms}$ (upper panel) and $t=10 \mathrm{~ms}$ (lower panel).

number of high-energy photons, considering the attenuation effect on the burst spectra at high energies. As the attenuation optical depth is highly sensitive to the bulk Lorentz factors of GRBs, we further constrain the properties of the Lorentz factors of GRBs. We find that to get a consistent LAT detection rate with the observed one, the distribution of Lorentz factors of the vast majority of GRBs should be in an appropriate range with 50-250 given variability time-scales of both $\Delta t=100$ and $10 \mathrm{~ms}$ for the prompt emissions of GRBs.

In this work, the Band function, an empirical function that has been fairly successful in fitting the spectra of most GRBs, is used to describe the GRB spectra. This empirical function may reflect the physics of the synchrotron emission (Zhang et al. 2016). There are some other functions available to fit the spectrum, such as the 'Comptonized' model and the smoothly broken power-law model which are preferred for fitting some GRBs (e.g. Kaneko et al. 2006; Kienlin et al. 2014; Bhat et al. 2016). Particularly, the 'Comptonized' model suggests an exponential cutoff in the spectrum at high energy and hence the extrapolated fluence at $100 \mathrm{MeV}$ is very low. However, we note that the uncertainty in the choice of the spectral function for GRB will not introduce much error in our results. This is because that GRBs that favour the 'Comptonized' model tend to have low fluence and/or soft spectrum above the peak energy. Thus, even if we extrapolate the fluences of these GRBs to high-energy energies with the 'Comptonized' model instead of the Band function, the predicted LAT detection rate will not change (see the Appendix for a detailed analysis).

\section{ACKNOWLEDGEMENTS}

We thank Song-Bo Zhang for providing the sample of Fermi/GBM GRBs and anonymous referee for the valuable comments that improve the work. This work is supported by the 973 program under grant 2014CB845800, and the Natural Science Foundation of China under grants 11625312 and 11851304 .

\section{REFERENCES}

Ackermann M. et al., 2011, ApJ, 729, 114

Ackermann M. et al., 2013, ApJS, 209, 11

Amati L. et al., 2002, A\&A, 390, 81

Band D. L. et al., 1993, ApJ, 413, 281

Band D. L. et al., 2009, ApJ, 701, 1673

Baring M. G., Harding A. K., 1997, ApJ, 491, 663

Bhat P. N. et al., 2016, ApJS, 223, 28

Fenimore E. E., Epstein R. I., Ho C., 1993, A\&AS, 97, 59

Ghirlanda G., Nava L., Ghisellini G., Firmani C., Cabrera J. I., 2008, MNRAS, 387, 319

Ghirlanda G., Nava L., Ghisellini G., Celotti A., Burlon D., Covina S., Melandri A., 2012, MNRAS, 420, 483

Goldstein A. et al., 2012, ApJS, 199, 19

Granot J., Cohen-Tanugi J., Silva E. D. C. E., 2008, ApJ, 677, 92

Guetta D., Pian E., Waxman E., 2011, A\&A, 525, A53

Hascoët R., Daigne F., Mochkovitch R., Vennin V., 2012, MNRAS, 421, 525

Kakuwa J., Murase K., Toma K., Inoue S., Yamazaki R., Ioka K., 2012, MNRAS, 425, 514

Kaneko Y., Preece R. D., Briggs M. S., Paciesas W. S., Meegan C. A., Band D. L., 2006, ApJS, 166, 298

Kienlin A. V. et al., 2014, ApJS, 211, 13

Krolik J. H., Pier E. A., 1991, ApJ, 373, 277

Liang E.-W., Yi S.-X., Zhang J., , Lü H.-J., Zhang B.-B., Zhang B., 2010, ApJ, 725, 2209

Lithwick Y., Sari R., 2001, ApJ, 555, 540

Liu R.-Y., Wang X.-Y., 2013, ApJ, 766, 73

Lloyd N. M., Petrosian V., Mallozzi R. S., 2000, ApJ, 534, 227

Lü J., Zou Y.-C., Lei W.-H., Zhang B., Wu Q., Wang D.-X., Liang E.-W., Lü H.-J., 2012, ApJ, 751, 49

Meegan C. A. et al., 2009, ApJ, 702, 791

Nava L., Ghirlanda G., Ghisellini G., Firmani C., 2008, MNRAS, 391, 639

Tang Q.-W.,, Peng F.-K., Wang X.-Y., Tam P.-H. T., 2015, ApJ, 806, 194

Vianello G., Gill R., Granot J., Omodei N., Cohen-Tanugi J., Longo F., 2017, ApJ, preprint (arXiv:1706.01481V)

Wanderman D., Piran T., 2010, MNRAS, 406, 1944

Woods E., Loeb A., 1995, ApJ, 453, 583

Yassine M., Piron F., Mochkovitch R., Daigne F., 2017, A\&A, 606, 93

Zhang B.-B., Uhm Z. L., Connaughton V., Briggs M. S., Zhang B., 2016, ApJ, 816, 72

Zou Y.-C., Fan Y.-Z., Piran T., 2011, ApJ, 726, 2

\section{APPENDIX A: SPECTRAL FUNCTION FOR THE PROMPT EMISSION OF GRBS: BAND VERSUS COMP}

We look up into the GBM GRB catalogue ${ }^{3}$ and collect a sample of 2099 GRBs with complete spectral information from 2008 August 14 to 2017 December 30. Among the sample, there are 1234 GRBs whose best-fitting model are labelled as 'COMP' (abbreviation for the 'Comptonized' model), 313 are labelled as 'BAND' (abbreviation for the Band function) or 'SBPL' (abbreviation for the smoothly broken power law), and the rest are labelled as 'PLAW'

\footnotetext{
${ }^{3}$ https://heasarc.gsfc.nasa.gov/W3Browse/fermi/fermigbrst.html
} 


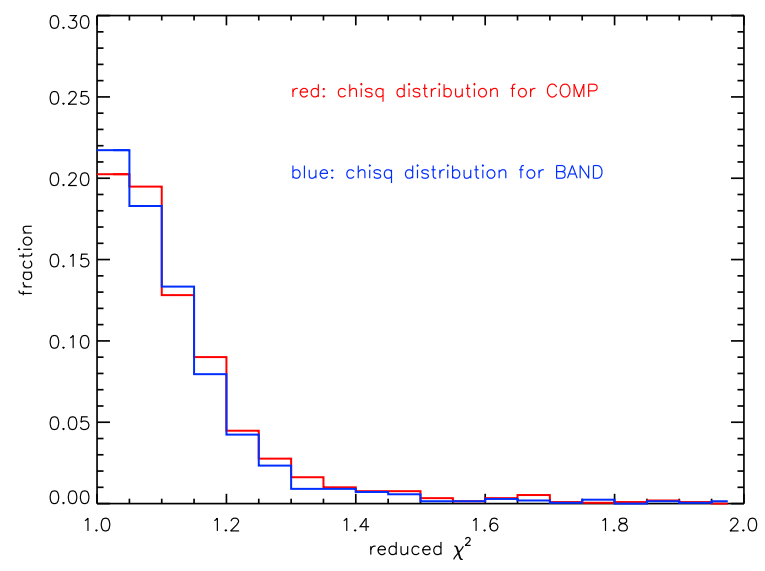

Figure A1. Distribution of the reduced $\chi^{2}$ when all the GRBs in the sample are fitted by COMP and BAND, respectively.

(abbreviation for power law). Extrapolating the flux to high energy with SBPL or PLAW does not result in significant difference from that with BAND, while extrapolating the flux with COMP leads to big difference, since it introduces an exponential cutoff in the high-energy spectrum. Thus, in what follows, we mainly discuss the difference between BAND and COMP and show that employing the BAND model as the intrinsic spectrum for all the simulated GRBs will not introduce much error in our results.

First, from the point of view of the $\chi^{2}$ test, BAND is preferred to COMP for the spectrum fitting. In the sample, there are 1425 GRBs with a smaller reduced $\chi^{2}$ value when fitted by BAND than fitted by COMP. This can be seen in the attached Fig. A1, in which we plot the respective distribution of the reduced $\chi^{2}$ value fitted by these two different models for all the GRBs in the sample. We can also see that, for the majority of GRBs, the reduced chi-square values are smaller than 1.5 for the BAND fitting, implying that BAND is nevertheless a quite acceptable model for the spectral fitting.

In the GBM GRB catalogue, the best-fitting model is determined by the Castor C-statistics instead of the $\chi^{2}$ test. The former one has a better performance than the latter one when there are a few counts in data bins, because the former one constructs a maximum-likelihood estimator $C$ based on the Poisson distribution of detected counts. Note that for a spectrum with many counts per bin, $C \rightarrow \chi^{2}$. Thus, for most GRBs which have a high fluence and a relatively hard spectrum above the peak energy, BAND is usually the best choice, or the improvement of the fitting with COMP with respect to that with BAND is not significant. To look into this issue, we show the 2D distribution of GRBs in the plane of $\beta$ (when GRBs are fitted by BAND) and the $1-1000 \mathrm{keV}$ fluence in Fig. A2. Most GRBs that are located at top-right region, the favoured region for LAT detection when extrapolating the spectrum up to $100 \mathrm{MeV}$, are best fitted by BAND or SBPL. Since the simulated GRB sample is consistent with the observed one in terms of both the $\beta$ distribution and the fluence distribution, we expect that the predicted LAT detection rate will not change even if we use COMP (instead of BAND) for the spectral extrapolation for those GRBs that are best fitted by COMP.

Besides, we calculate the LAT detection rate of the simulated GRB sample with the same method introduced in Section 2 and 3, but using COMP instead of BAND. It results in null detection of LAT, suggesting that COMP cannot describe all the GRBs.

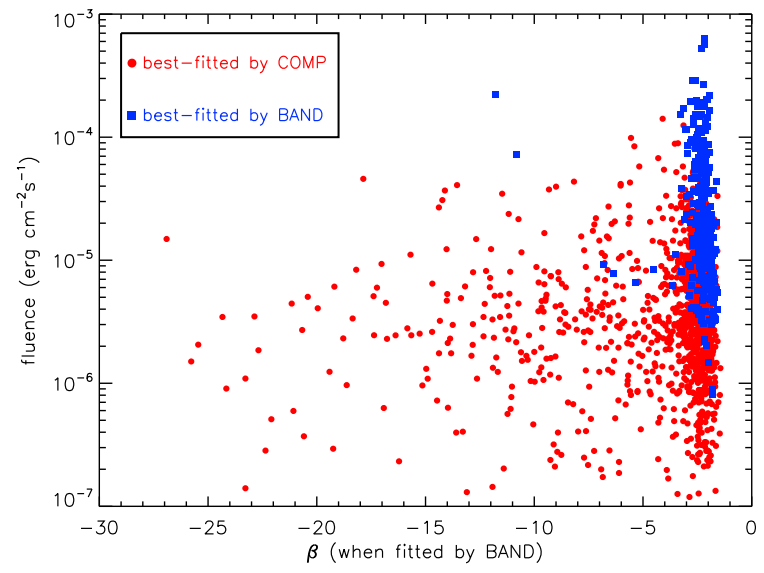

Figure A2. 2D distribution of GRBs in the $\beta$-fluenceplane. Red filled circles represent GRBs best fitted by COMP while blue filled squares represent GRBs best fitted by BAND. 
Table A1. Spectral properties of 72 long Fermi/GBM GRBs with measured redshifts, which are taken from Jochen Greiner's collection ${ }^{a}$.

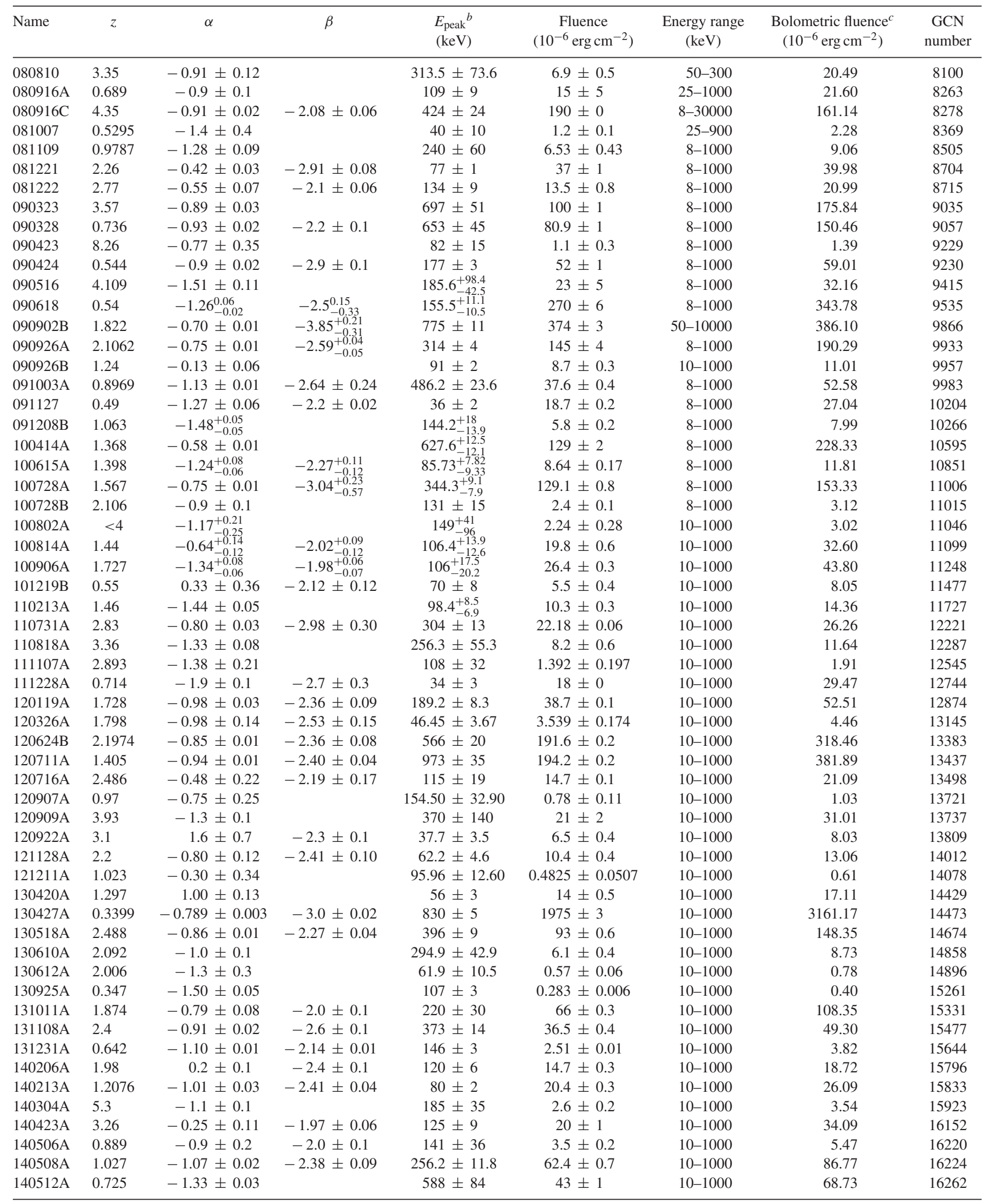


Table A1. - continued

\begin{tabular}{|c|c|c|c|c|c|c|c|c|}
\hline Name & $z$ & $\alpha$ & $\beta$ & $\begin{array}{l}E_{\text {peak }} b \\
(\mathrm{keV})\end{array}$ & $\begin{array}{c}\text { Fluence } \\
\left(10^{-6} \mathrm{erg} \mathrm{cm}^{-2}\right)\end{array}$ & $\begin{array}{l}\text { Energy range } \\
(\mathrm{keV})\end{array}$ & $\begin{array}{l}\text { Bolometric fluence } \\
\quad\left(10^{-6} \mathrm{erg} \mathrm{cm}^{-2}\right)\end{array}$ & $\begin{array}{c}\text { GCN } \\
\text { number }\end{array}$ \\
\hline 140606B & 0.384 & $-1.22 \pm 0.04$ & & $473.00 \pm 82.60$ & $9.018 \pm 0.369$ & $10-1000$ & 13.88 & 16363 \\
\hline $140623 \mathrm{~A}$ & 1.92 & $-1.46 \pm 0.1$ & & $350_{-100}^{+220}$ & $6.8 \pm 0.6$ & $10-1000$ & 10.07 & 16450 \\
\hline $140703 \mathrm{~A}$ & 3.14 & $-1.10 \pm 0.06$ & & $177 \pm 14$ & $0.74 \pm 0.04$ & $10-1000$ & 1.00 & 16512 \\
\hline $140801 \mathrm{~A}$ & 1.32 & $-0.40 \pm 0.04$ & $-3.6 \pm 0.4$ & $122 \pm 3$ & $12.2 \pm 0.1$ & $10-1000$ & 12.71 & 16658 \\
\hline 141004A & 0.573 & $-1.3 \pm 0.1$ & & $147 \pm 28$ & $2.2 \pm 0.2$ & $10-1000$ & 3.00 & 16900 \\
\hline $141220 \mathrm{~A}$ & 1.3195 & $-0.80 \pm 0.05$ & & $180 \pm 9$ & $5.23 \pm 0.15$ & $8-1000$ & 7.00 & 17205 \\
\hline $141221 \mathrm{~A}$ & 1.452 & $-1.07 \pm 0.13$ & & $152.4 \pm 28.5$ & $3.4 \pm 0.4$ & $8-1000$ & 4.50 & 17216 \\
\hline $141225 \mathrm{~A}$ & 0.915 & $-0.35 \pm 0.17$ & $-2.0 \pm 0.1$ & $187 \pm 29$ & $6.5 \pm 0.3$ & $10-1000$ & 10.66 & 17241 \\
\hline 150301B & 1.5169 & $-1.1 \pm 0.1$ & $-2.0 \pm 0.2$ & $180 \pm 38$ & $4.4 \pm 0.2$ & $10-1000$ & 6.52 & 17525 \\
\hline 150314A & 1.758 & $-0.60 \pm 0.01$ & $-2.32 \pm 0.04$ & $311 \pm 5$ & $85.3 \pm 0.4$ & $10-1000$ & 128.06 & 17579 \\
\hline
\end{tabular}

Notes: ${ }^{a}$ http://www.mpe.mpg.de/ jcg/grbgen.html

${ }^{b}$ In the observer's frame.

${ }^{c}$ In the energy range of $1-10000 \mathrm{keV}$.

This paper has been typeset from a $\mathrm{T}_{\mathrm{E}} \mathrm{X} / \mathrm{LT} \mathrm{E} \mathrm{X}$ file prepared by the author. 